\title{
TC-99M MIBI SPECT AND CT ASSESSMENT OF RECURRENT ACOUSTIC NEUROMA: A CASE REPORT AND REVIEW OF THE LITERATURE
}

\author{
A. Kaprelyan, N. Deleva, ${ }^{1}$ P. Bochev, ${ }^{1}$ A. Klissarova, ${ }^{1}$ B. Balev, ${ }^{2}$ G. Kuchukov

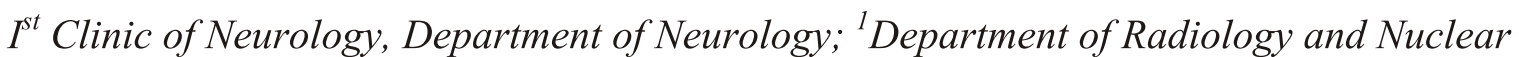 \\ medicine; ${ }^{2}$ Department of Neurosurgery, Prof. Paraskev Stoyanov Medical University of Varna
}

\begin{abstract}
Acoustic neuromas are non-malignant tumors of the eighth cranial nerve that comprise approximately $6 \%$ of all intracranial neoplasms and about $85 \%$ of CPA tumors. Early diagnosis is based on medical history of unilateral hearing loss, tinnitus and unsteadiness, audiometric tests, and MRI or CT findings. Although recurrence is uncommon after tumor removal, postoperative neuroimaging is recommended in patients with clinical deterioration. We report a case of 57-years male presented with a progressive headache, unsteadiness, right-sided facial and limbs weakness. Medical history revealed removal of an acoustic neuroma fourteen years ago. On neurological examination he had right-sided facial numbness and palsy, ipsilateral hypacusis, horizontal nystagmus, mild muscle hypotonia and also limbs ataxia. CT scans visualized a contrast-enhanced hyperdense lesion located in the right CPA. Tc-99m MIBI brain SPECT demonstrated an increased tracer accumulation in accordance with the tumor relapse. Our own findings and review of the literature suggest that Tc-99m MIBI SPECT may be useful for the detection of recurrent posterior fossa tumors.
\end{abstract}

Keywords: acoustic neuroma, tumor recurrence, Tc-99m MIBI SPECT

\section{INTRODUCTION}

Acoustic neuromas, also known as vestibular schwannomas, are non-malignant tumors that arise from the Schwann cell sheath of the 8th cranial nerve $(7,14)$. They comprise approximately $6 \%$ of all intracranial neoplasms and about $85 \%$ of cerebellopontine angle (CPA) tumors $(12,18,20)$. The most frequent symptoms are hearing loss, tinnitus, and unsteadiness. The diagnosis is based on medical history, different audiometric tests, and contrast-enhanced computed tomography (CT) or magnetic resonance imaging (MRI) $(5,7,20)$. Because the hearing loss is the most frequent symptom, the conventional audiometry is considered to be the best diagnostic test (12, 14,17,18). CT scanning with contrast can demonstrate tumors larger than $1.5 \mathrm{~cm}$ while gadolinium-enhanced MRI is the definitive diagnostic test for acoustic neuromas as small as $1-2 \mathrm{~mm}$ in diameter $(5,13,18)$. The differential diagnosis includes meningiomas, facial nerve neuromas, vascular tumors, lipomas or cerebral metastases. Microsurgery and radiotherapy are most usually applied $(5,9,18,25)$.

Although tumor recurrence is uncommon, neuroimaging is recommended in patients with clinical deterioration after tumor removal. Data exist that radiological imaging plays

\footnotetext{
Address for correspondence:

Ara Kaprelyan, Dept of Neurology, Prof. Paraskev Stoyanov Medical University of Varna, 55 M. Drinov Str., 9002 Varna, BULGARIA,

E-mail: arakapri07@yahoo.co.uk
}

an important role in assessment of intracranial lesions, but sometimes CT and MRI diagnosis is difficult $(12,13,23)$. Accordingly, the new functional technique such as single photon emission computed tomography (SPECT) is a promising alternative $(1,10,21,23,24)$. Previous reports suggest that Tc-99m MIBI is a small lipophilic radioligand that is preferentially trapped in mitochondria $(2,3,15,19)$. Thus, as a result of high mitochondrial activity in tumors it accumulates predominantly in neoplastic tissue compared to normal one $(1,2,10,22,24)$. Several studies demonstrate its usefulness for the primary diagnosis, assessment of therapeutic response, and detection of tumor relapse $(3,4,6$, $11,15,19,23,24)$.

\section{CASE REPORT}

A 57-years male was admitted to our clinic with progressive unsteadiness, intense headache, facial numbness, and weakness in right extremities. On neurological examination he had right-sided facial hypesthesia and palsy (lagophthalm), ipsilateral hypacusis, horizontal nystagmus, limbs ataxia, and also a mild muscle hypotonia. CT scans visualized a postoperative cyst in the right cerebellar hemisphere and a contrast-enhanced, hyperdense, two-component lesion (respectively, $13 / 26 \mathrm{~mm}$ and $11 / 19 \mathrm{~mm}$ ) located in the right CPA (fig. 1.). Tc-99m MIBI brain SPECT demonstrated an increased tracer accumulation in the same region due to tumor recurrence (fig. 2.). 


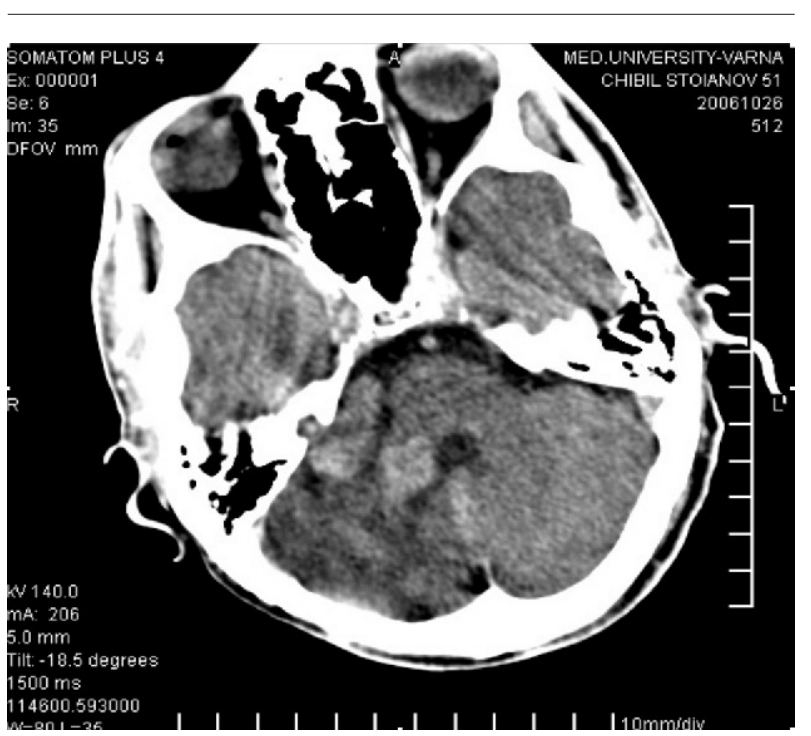

Fig. 1. CT scan shows a recurrent hyperdense, two-component lesion in the right $C P A$. located in the right CPA, perifocal edema, as well as dislocation of fourth ventricle and aqueduct. The tumor removal with a suboccipital (retrosigmoid) approach was thought to be complete. An acoustic neuroma was confirmed by histopathology. During the next fourteen years the patient remained clinically stable.

\section{DISCUSSION}

Acoustic neuromas are the most common CPA tumors that account for approximately $6 \%$ of all cerebral neoplasms (7, $14,20)$. They are relatively uncommon and affect approximately 1 in 100,000 people per year. The majority of cases is sporadic, unilateral, and usually occurs in adults $(12,18)$. The most common symptoms involve hearing loss, tinnitus, and dizziness. The clinical findings concerning our patient's age (43 years) and presentation (headache, hearing impairment, and imbalance) are in accordance with these previous observations. Especially, unsteadiness, fa-

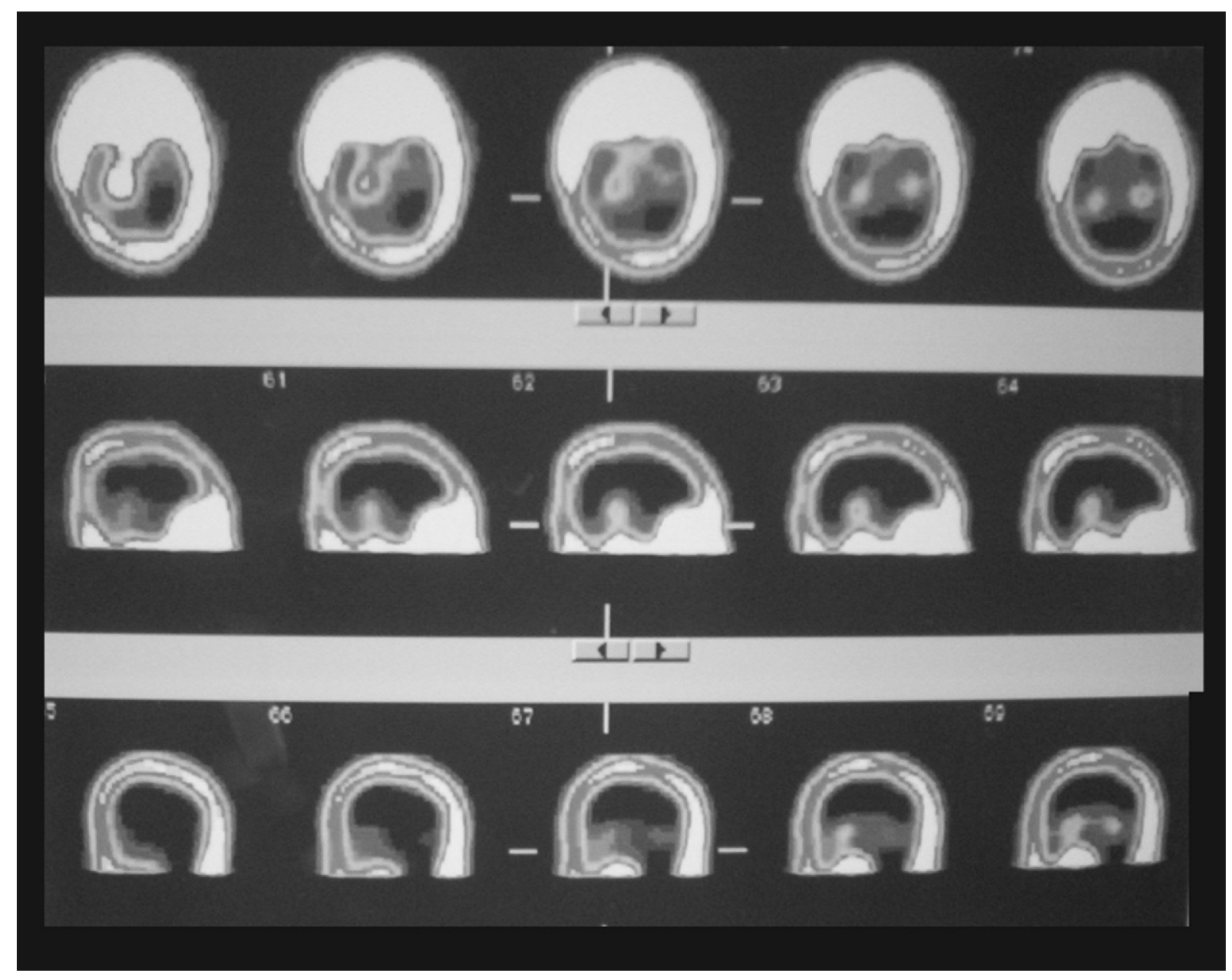

Fig. 2. Tc-99m MIBI brain SPECT demonstrates an increased tracer accumulation in the right CPA.

Medical history revealed an onset of unilateral hearing impairment, headache, imbalance, weakness in right extremities, and a speech disturbance at the age of 43 years. The primary CT scans showed a large tumor mass $(32 / 52 \mathrm{~mm})$ cial sensory disturbances and hearing loss are more common with large tumors $(14,17,18)$.

Diagnosis of acoustic neuromas includes audiometry, auditory brainstem responses, and neuroimaging $(5,17,20)$. 
Several studies report that contrast-enhanced CT scans and MRI are the most sensitive methods for the early detection of acoustic neuromas, evaluation of their size and location, and also planning of surgical approach $(5,12,13,18,25)$. Our radiological findings of a large tumor mass support the opinion that CT scanning with contrast succeeds to detect a medium-size or large CPA tumor.

The various treatment modalities include conservative management, surgery, gamma-knife procedure or stereotactic radiotherapy $(5,9,25)$. About quarter of all acoustic neuromas are treated with medical management that consists of neurological status monitoring, serial audiometry, and periodic neuroimaging $(18,20)$. About half of all acoustic neuromas are presently treated with surgery $(5,17,25)$. In accordance with these reports, our patient underwent craniotomy and gross total resection with suboccipital approach. His initial right-sided hearing impairment and cerebellar symptoms had significantly improved postoperatively in correspondence with previous observations $(5,12,14,20)$.

Data exist that recurrence rate is $5-10 \%$ or lower after acoustic tumor removal $(5,9,20)$. It is established that the vast majority of recurrences follow suboccipital removal. Although tumor relapse is uncommon, we support the opinion that any postoperative clinical deterioration independent of time of manifestation may be a signal for a tumor recurrence $(6,12,14,17)$. Thus, the appearance of progressive unsteadiness, intense headache, altered sensation to the face, facial and right extremities weakness fourteen years after acoustic neuroma removal supposed in our patient a tumor growth and required a control neuroimaging.

Although the traditional neuroimagery plays an important role in evaluation of cerebral neoplasms, it has been shown that the new functional methods such as brain SPECT may improve their diagnosis $(2,19,21,23,24)$. From this point of view, preliminary data suggest that different supratentorial and CPA tumors can be successfully visualized using cerebral tomoscintigraphy with Tc-99m MIBI $(1,3,8,11,15$, 16). In our patient a brain SPECT was performed after i.v. injection of $20 \mathrm{mCi}$ Tc-99m MIBI. The increased tracer uptake in the right CPA was in correlation with the positive $\mathrm{CT}$ scans demonstrating the acoustic neuroma relapse. Accordingly, our previous and present findings correspond with the observations of several authors that Tc-99m MIBI may be especially useful for the detection of recurrent tumors in patients who have undergone neurosurgery $(4,6,8$, $10,11,22)$.

\section{CONCLUSION}

Based on the literature review and our own results we suggest that in patients with clinical deterioration after tumor removal both anatomical and functional neuroimaging is necessary to detect a possible tumor relapse. Accordingly, Tc-99m MIBI SPECT is a modern diagnostic technique which may be useful in diagnosis and follow-up of recurrent posterior fossa tumors.

\section{REFERENCES:}

1. Ak I., Gulbas Z., Altinel F., Vardareli E. Tc-99m MIBI uptake and its relation to the proliferative potential of brain tumors. Clin. Nucl. Med., 1, 2003, 28, 29-33.

2. Bagni B., Pinna L., Tamarozzi R., Cattaruzzi E., et al. SPET imaging of intracranial tumours with 99Tcm-sestamibi. Nucl. Med. Commun., 1995, 4, 16, 258-64.

3. Baldari S., Pecorella G., Cosentino S., Minutoli F. Investigation of brain tumours with (99m) Tc-MIBI SPET. Q. J. Nucl. Med., 4, 2002, 46, 336-345.

4. Barai S., Bandopadhayaya G., Julka P., et al. Evaluation of single photon emission computerised tomography (SPECT) using Tc99mtetrofosmin as a diagnostic modality for recurrent posterior fossa tumors. J. Postgrad Med., 2003, 49, 316-21.

5. Barker F., Carter B., Ojemann R., Jyung R., Poe D., McKenna M. Surgical excision of acoustic neuroma: patient outcome and provider caseload. $\mathrm{La}$ ryngoscope, 8, 2003, 113, 1332-1343.

6. Deleva N., Kaprelyan A., Bochev P., Klissarova A., Dimitrov I. SPECT imaging of brain lesions associated with refractory epilepsy. Scri. Sci. Med., 39, 2007, 1, 67-70.

7. Evans, D. G., A. Moran, et al. Incidence of vestibular schwannoma and neurofibromatosis 2 in the North West of England over a 10-year period: higher incidence than previously thought. Otol. Neurotol., 1, 2005, 26, 93-97.

8. Goethals I., De Winter O., Dierckx R., Annovazzi A., Signore A., Van de Wiele C. False-negative Tc-99m MIBI scintigraphy in histopathologically proved recurrent high-grade oligodendroglioma. Clin. Nucl. Med., 4, 2003, 28, 299-301.

9. Jarvis S., Davis A., Lang D., Fugleholm K. Surgery for Vestibular Neuroma: Resection, residue, and recurrence. Skull Base, 2005, 15.

10. Jeune F., Dubois F., Blond S., Steinling M. Sestamibi technetium-99m brain single-photon emission computed tomography to identify recurrent glioma in adults: 201 studies. J. Neurooncol. 2, 2006, 77, 177-183.

11. Kaprelyan A. Tc-99m MIBI brain SPECT imaging of intracranial tumors. J. Neuro-Oncol., Oct. 2006, 372.

12. Lee W., Isaacson J. Postoperative imaging and follow-up of vestibular schwannomas. Otol. Neurotol., 1, 2005, 26, 102-104.

13. Matthews P., Wylezinska M., Cadoux-Hudson T. Novel approaches to imaging brain tumors. Hematology/oncology Cl. N. Am., 15, 2001, 4.

14. Neff B., Willcox T., Sataloff R. Intralabyrinthine schwannomas. Otol. Neurotol., 2003, 24, 299-307. 
15. Park C., Kim S., Zhang J., McEwan J., Intenzo C. ${ }^{99 \mathrm{~m}} \mathrm{Tc}-\mathrm{MIBI}$ brain SPET of an acoustic shwannoma. Clin. Nucl. Med., 1994, 19, 152-154.

16. Park C., Sataloff R., Richard M., Zhang J., Kim S. Tc-99m MIBI brain SPECT of cerebellopontine angle tumors. Clin. Nucl. Med., 5, 1996, 21, 375-378.

17. Piazza F., et al. Management of acoustic neuromas in the elderly: Retrospective study. ENT J., May 2003, 374-378.

18. Perry B., Gantz B., Rubinstein J. Acoustic neuromas in the elderly. Otol. Neurotol., 2001, 22, 389-391.

19. Prigent-Le Jeune F., Dubois F., Perez S., Blond S., Steinling M. Technetium-99m sestamibi brain SPECT in the follow-up of glioma for evaluation of response to chemotherapy: first results. Eur. J. Nucl. Med. Mol. Imaging. 5, 2004, 31, 714-719.

20. Roland P. Skull Base, Acoustic Neuroma (Vestibular Schwannoma). eMedecine, 2006.
21. Single Photon Emission Computed Tomography (SPECT). Clinical Policy Bulletins, 0376, 2006.

22. Shih W., Lee J., Milan P. Discordant technetium-99m-MIBI and technetium-99m-HMPAO uptake of recurrent occipital meningioma on brain SPECT images. J. Nucl. Med., 5, 1997, 38, 828.

23. Sygitowicz M., Lass P., Lyczak P., Stepien-Kocmiel E., Taraszewska M., Bandurski T. Tc-99m-MIBI and Tc-99m-HMPAO accumulation in primary and metastatic brain tumors assessed by brain SPECT. Neurol. Neurochir. Pol., 5, 1998, 32, 1099-1106.

24. Tomura N., Hirano H., Watanabe O., et al. Preliminary results with technetium-99m MIBI SPECT imaging in patients with brain tumors: correlation with histological and neuroradiological diagnoses and therapeutic response. Comput. Med. Imaging Graph., 5, 1997, 21, 293-298.

25. Wackym P., et al. Gamma knife radiosurgery for acoustic neuromas performed by a neurootologist: early experiences and outcomes. Otol. Neurotol., 2004, 25, 752-761. 